\title{
Anormalidades Cineangiocoronariográficas em Pacientes com Isquemia Miocárdica à Cintilografia Perfusional e Coronárias sem Lesões Obstrutivas: Estudo Descritivo
}

\author{
Luiz de Castro Bastos ${ }^{1}$, Cláudio Leinig Pereira da Cunha²
}

\section{RESUMO}

Objetivo: Identificar as anormalidades angiográficas encontradas nos pacientes com Cintilografia de Perfusão Miocárdica (CMP) positiva para isquemia, nos quais a Cineangiocoronariografia (CINE) não revelou lesão coronariana obstrutiva. Método: Foram avaliados, retrospectivamente, 105 pacientes, sendo $61(58 \%)$ do sexo feminino e $44(42 \%)$ do masculino. A idade variou de 31 a 78 anos. As anormalidades foram divididas em nove categorias: (1) Circulação Coronária sem lesões obstrutivas (Normal), (2) Circulação Coronária com Tortuosidades, (3) Circulação Coronária com Espasmo, (4) Ponte Miocárdica, (5) Prolapso da Valva Mitral, (6) Circulação Coronária com Afilamento, (7) Anomalia Congênita Coronária - Fístula, (8) Ventrículo esquerdo hipertrófico e (9) Cardiomiopatia Dilatada. Resultados: Foram encontradas 150 anormalidades cineangiocoronariográficas, isoladas ou em associação, assim distribuídas: 98 nas artérias coronárias, 51 no ventrículo esquerdo e uma na valva mitral. Conclusões: Conclui-se que estas anormalidades, quando presentes nos pacientes sem lesões obstrutivas coronarianas, podem ser causa de CPM "falso-positivas".

DESCRITORES: Angiografia coronária. Cintilografia. Coronariopatia, radiografia. Cateterismo cardíaco.
A s doenças cardiovasculares (DCV) são reconhecidas pela Organização Mundial de Saúde como a principal causa de mortalidade no mundo ocidental ${ }^{1}$. No Brasil ${ }^{2,3}$, elas são a principal causa de morte, vitimando, anualmente, 300.000 brasileiros, isto é, 34 por hora ou um óbito a cada dois minutos. Isto

\footnotetext{
'Digicor - Cardiologia e Angiologia Digital Serviço de Hemodinâmica do Hospital Policlínica Cascavel, PR.

2 Universidade Federal do Paraná, PR.

Correspondência: Luiz de Castro Bastos. R. Carlos de Carvalho, 2982

- Bairro Ciro Nardi - Cascavel - PR - CEP 85802-090

Recebido em: 22/11/2006 - Aceito em: 14/2/2007
}

\section{SUMMARY}

Coronary Artery Angiograms in Patients with Positive Myocardium Scintigram and Normal Coronary Artery Angiograms: a Descriptive Study

Objetive: To identify pathologic angiography findings in patients with positive myocardium scintigram and normal coronary artery angiogram. Methods: One hundred and five (105) patients were assessed retrospectively, being 61 females $(58 \%)$ and 44 males $(42 \%)$, in the 31-78-year-old range. Abnormalities were divided into 9 categories following angiographic findings: (1) coronary circulation without obstructive lesions; (2) tortuous coronary arteries; (3) coronary circulation with spasm; (4) myocardial bridge, (5) mitral valve prolapse; (6) coronary circulation with diffuse luminal narrowing; (7) coronary fistula; (8) left ventricular hypertrophy; and (9) dilated cardiomyopathy. Results: One hundred fifty (150) angiographic abnormalities were found, either isolatedly or in combination: 98 patients with abnormal coronary findings, 51 with abnormal left ventricle findings, and one abnormal mitral valve finding. Conclusion: In the presence of normal coronary angiograms those abnormalities could be responsible for "false-positive" myocardial scintigrams.

DESCRIPTORS: Coronary angiography. Radionuclide imaging. Coronary disease, radiography. Heart catheterization.

representa a maior causa de gastos com a assistência médica: $16,22 \%$ do total $^{4}$.

A partir destes dados, observa-se a importância de se adotar medidas preventivas objetivando a redução de tão desfavorável realidade. Os mesmos demonstram a importância e a necessidade da realização de testes não invasivos, nestes indivíduos, que permitam detectar a presença de doença arterial coronária obstrutiva (DAC) e, assim, encaminhá-los à cineangiocoronariografia (CINE).

Desde sua introdução na prática clínica, em $1964^{5}$, e até mais recentemente ${ }^{6}$, a Cintilografia de Perfusão Miocárdica (CPM) é considerada de indicação prioritária 
Bastos LC, Cunha CLP. Anormalidades Cineangiocoronariográficas em Pacientes com Isquemia Miocárdica à Cintilografia Perfusional e Coronárias sem Lesões Obstrutivas: Estudo Descritivo. Rev Bras Cardiol Invas 2007; 15(1): 52-60.

para pacientes com suspeita de DAC, sendo preferencialmente associada ao estresse, físico ou farmacológico. Segundo a I Diretriz da Sociedade Brasileira de Cardiologia sobre Cardiologia Nuclear ${ }^{7}$, a CPM é especialmente indicada em pacientes nos quais haja dificuldades para a interpretação adequada do eletrocardiograma (ECG) de esforço.

Em busca de aprimorar a definição deste método não invasivo, foram introduzidos, na prática clínica, programas de computador que permitiram a realização da CPM em cortes tomográficos ${ }^{8}$, incrementando a capacidade de identificar doença coronária em números superiores a $90 \%$ dos casos. Isto transformou a CPM no método não invasivo mais sensível ${ }^{9}$, apresentando especificidade de $93 \%{ }^{10}$.

São conhecidas diversas condições clínicas responsáveis por testes positivos ${ }^{11}$, na ausência de DAC, tais como:

a) cardiomiopatia;

b) hipertrofia ventricular esquerda (HVE) - inclusive a fisiológica do atleta;

C) prolapso da valva mitral (PVM);

d) BRE;

e) anormalidade vasorreguladora: espasmo, tortuosidades e afilamento desproporcionado;

f) ponte miocárdica;

g) artefatos de atenuação como a mama esquerda em mulheres;

h) síndrome " $X$ ".

Apesar de sua alta sensibilidade, a CPM ainda é um método que não identifica $100 \%$ dos doentes quando ela é anormal e não ratifica 100\% dos indivíduos não doentes quando ela é normal ${ }^{12}$. Desta forma, a CINE tornou-se o padrão-ouro. Este padrão é tão reconhecido no meio cardiológico que raramente comenta-se a possibilidade de ocorrerem erros de diagnóstico. Porém, a despeito do seu grande desenvolvimento, existem situações, embora raras, em que esta não identifica lesões ateroscleróticas, tais como: projeções inadequadas, superposição do diafragma, superposição de vasos, lesões excêntricas, número limitado de projeções e diferenças inter e intra-observadores ${ }^{13}$. Apesar destas limitações, a CINE tem sido utilizada para a avaliação da precisão diagnóstica dos métodos que estudam a isquemia miocárdica ${ }^{13}$. Isto se deve, provavelmente, à carência de outro método mais objetivo e preciso na identificação de DAC. Entretanto, deve-se estar atento à outra limitação deste método que é a possibilidade de subestimação ou superestimação das lesões coronarianas ${ }^{13,14}$.

Na prática clínica, a função principal da CINE é detectar a presença ou não de DAC. Na análise de suas imagens, há uma tendência de se menosprezar lesões abaixo de $50 \%$, correndo-se o risco de subestimá$\operatorname{las}^{15}$. Entretanto, vários autores ${ }^{16-18}$ têm demonstrado a aplicabilidade e confiança do método de quantificação visual, sendo o mesmo aceito e realizado na maioria dos centros médicos.

Assim, o objetivo deste trabalho é identificar as anormalidades angiográficas encontradas na CINE dos pacientes com CPM positiva, nos quais não se identificou lesão coronariana obstrutiva.

\section{MÉTODO}

Estudo retrospectivo, no qual foram incluídos pacientes com sinais de isquemia miocárdica detectada na CPM, encaminhados a CINE para confirmação de DAC, independentemente da idade, sexo e raça, cujo resultado não demonstrou lesão obstrutiva coronariana. Foram excluídos todos os pacientes que apresentassem na CINE qualquer tipo de lesão obstrutiva, independente do seu grau de obstrução.

No período de fevereiro de 1999 a julho de 2003, 105 pacientes foram submetidos a CINE, no Serviço de Hemodinâmica do Hospital Policlínica Cascavel Digicor - Cardiologia e Angiologia Digital, para confirmação de DAC, sendo 61 pacientes (58\%) do sexo feminino e 44 (42\%) do masculino. A idade variou de 31 a 78 anos, média de 55,32 \pm 10,36 anos. Os dados referentes ao perfil geral dos pacientes encontram-se na Tabela 1.

Foram utilizadas as seguintes definições angiográficas:

1. Circulação coronariana sem lesões obstrutivas (NORMAL): quando as imagens obtidas nas diversas projeções não mostrassem nenhuma alteração da árvore arterial $^{19}$;

2. Circulação coronariana com tortuosidades: quando as imagens obtidas evidenciassem trajetos tortuosos, segundo os critérios do índice de tortuosidade de Macruz et al. ${ }^{20}$;

TABELA 1

Perfil geral dos pacientes

\begin{tabular}{lccc}
\hline Parâmetros & \multicolumn{2}{c}{ Sexo } & $\begin{array}{c}\text { Total de } \\
\text { Pacientes: } \\
\text { n }=\mathbf{1 0 5}\end{array}$ \\
\cline { 2 - 3 } & Masculino & Feminino & \\
\hline Pacientes & 44 & 61 & \\
Idade Máxima & 76 & 78 & \\
Idade Mínima & 31 & 32 & \\
Média & 52,25 & 57,64 & 55,32 \\
Desvio-padrão & 11,81 & 8,62 & 10,36 \\
\hline
\end{tabular}


Bastos LC, Cunha CLP. Anormalidades Cineangiocoronariográficas em Pacientes com Isquemia Miocárdica à Cintilografia Perfusional e Coronárias sem Lesões Obstrutivas: Estudo Descritivo. Rev Bras Cardiol Invas 2007; 15(1): 52-60.

3. Circulação coronariana com espasmo: quando ocorresse diminuição transitória da luz do vaso, desaparecendo após a utilização de vasodilatador intracoronário ${ }^{21}$;

4. Ponte miocárdica: quando ocorresse estreitamento transitório da luz da artéria, evidenciado durante a sístole, segundo os critérios de Carvalho et al. ${ }^{22}$;

5. Prolapso da valva mitral: quando ocorresse o deslocamento de um dos folhetos para além do nível do anel da valva mitral ${ }^{23}$;

6. Circulação coronariana com afilamento: quando ocorresse diminuição do calibre arterial maior do que a condicionada pela saída normal dos ramos arteriais ${ }^{20}$;

7. Anomalia congênita coronária-fístula: quando houvesse uma comunicação anômala entre uma artéria e uma cavidade, ou outra artéria, ou uma veia ${ }^{24}$;

8. Ventrículo esquerdo hipertrófico: imagem de aumento da massa muscular do ventrículo esquerdo, provocando um distanciamento acentuado entre as imagens das artérias coronárias e o endocárdio, na sístole ${ }^{25}$;

9. Miocardiopatia dilatada: quando a imagem adquirida na fase final da sístole demonstrasse uma hipocinesia difusa +++ ou $++++/ 4+{ }^{25}$.

Todos os procedimentos foram realizados em um aparelho de hemodinâmica da Siemens, modelo Coroskop-Hicor, fabricado na Alemanha, em 1997, compu- tadorizado, com sistema digital e subtração de imagens, dotado de um sistema de arquivo e de análise das imagens em compact disc (CD).

Todos os pacientes com CPM positiva foram submetidos a CINE, após a autorização por escrito. As técnicas empregadas foram as clássicas descritas por Sones ${ }^{26}$, quando a via de acesso foi a braquial, ou por Judkins ${ }^{27}$, quando a via de acesso foi a femoral.

Em todas as cenas tomadas do equipamento de CINE, foram utilizadas filmagens de 15 quadros por segundo, gravadas em CD, para posterior análise e arquivo.

Todos os procedimentos ocorreram sem complicações, havendo liberação dos pacientes para casa com um mínimo de 3 e um máximo de 6 horas após os mesmos, quando a via de acesso foi a braquial ou a femoral, respectivamente.

\section{RESULTADOS}

Considerando-se as definições adotadas na seção Método, foram encontradas 17 anormalidades na CINE, isoladas ou em associação, com as respectivas freqüências em relação ao sexo e ao número de casos, descritas na Tabela 2. As Figuras 1 e 2 representam dois exemplos fotográficos daquelas anormalidades, descrevendo-se, em ambas, os achados da CPM, com seus correspondentes achados na CINE.

Excetuando-se a circulação coronariana sem lesões

TABELA 2

Anormalidades angiográficas

\begin{tabular}{|c|c|c|c|c|}
\hline \multirow[t]{2}{*}{ № } & \multirow[t]{2}{*}{ Resultados } & \multicolumn{2}{|c|}{ Sexo } & \multirow{2}{*}{$\begin{array}{c}\text { Total } \\
\text { de caso }\end{array}$} \\
\hline & & Masculino & Feminino & \\
\hline 1 & Ventrículo Esquerdo Hipertrófico + Tortuosidade & 5 & 19 & 24 \\
\hline 2 & Tortuosidades & 4 & 15 & 19 \\
\hline 3 & Normal & 11 & 6 & 17 \\
\hline 4 & Ventrículo Esquerdo Hipertrófico & 5 & 4 & 9 \\
\hline 5 & Ponte Miocárdica & 5 & 1 & 6 \\
\hline 6 & Tortuosidades + Afilamento & 3 & 3 & 6 \\
\hline 7 & Ventrículo Esquerdo Hipertrófico + Tortuosidades + Afilamento & 2 & 4 & 6 \\
\hline 8 & Ventrículo Esquerdo Hipertrófico + Tortuosidades + Ponte Miocárdica & 3 & 2 & 5 \\
\hline 9 & Ponte Miocárdica + Tortuosidades & 1 & 2 & 3 \\
\hline 10 & Cardiomiopatia Dilatada & 1 & 1 & 2 \\
\hline 11 & Ventrículo Esquerdo Hipertrófico + Afilamento & - & 2 & 2 \\
\hline 12 & Cardiomiopatia Dilatada + Tortuosidades & - & 1 & 1 \\
\hline 13 & Ventrículo Esquerdo Hipertrófico + Anomalia Congênita Coronária & 1 & - & 1 \\
\hline 14 & Prolapso de Valva Mitral & 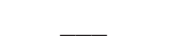 & 1 & 1 \\
\hline 15 & Ventrículo Esquerdo Hipertrófico + Ponte Miocárdica & 1 & - & 1 \\
\hline 16 & Ponte Miocárdica + Espasmo & 1 & - & 1 \\
\hline 17 & Tortuosidades + Espasmo & 1 & - & 1 \\
\hline \multicolumn{2}{|c|}{ T O T A L } & 44 & 61 & 105 \\
\hline
\end{tabular}


Bastos LC, Cunha CLP. Anormalidades Cineangiocoronariográficas em Pacientes com Isquemia Miocárdica à Cintilografia Perfusional e Coronárias sem Lesões Obstrutivas: Estudo Descritivo. Rev Bras Cardiol Invas 2007; 15(1): 52-60.

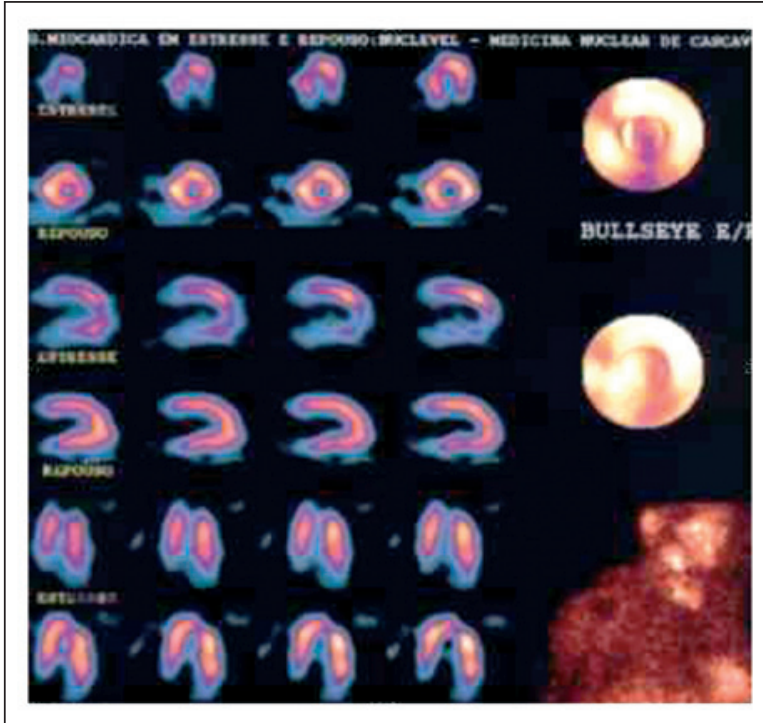

Figura 1 - Caso № 21 - E.C.S cat 8298 - 62 anos - Fem. - Conclusão: Cintilografia miocárdica perfusional com Tc 99m-Sestamibi, por técnica tomográfica, compatível com hipocaptação reversível em região ântero-septal do VE (alta probabilidade de isquemia miocárdica).

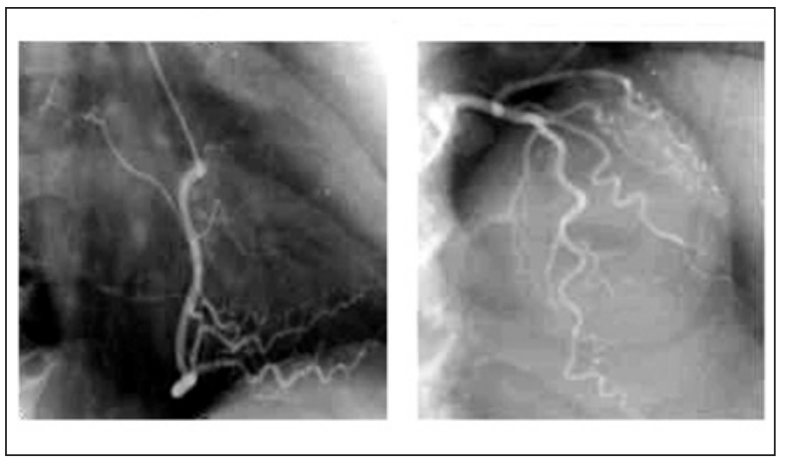

Figura 2 - Caso № 54 - N. K. M. - Cat. 11190 e CM - 32 anos - Fem. - Circulação coronária com Tortuosidades. obstrutivas (NORMAL), todos os casos de anormalidades encontradas naquelas 17 categorias foram somados, em ordem decrescente. Esta somatória foi feita enquadrando-se aquelas anormalidades em outras 8 definições angiográficas, obtendo-se 150 anormalidades, discriminadas na Tabela 3.

Esta tabela mostra, então, a seguinte freqüência de cada definição angiográfica em relação ao:

- Sexo feminino:

a) Tortuosidades;

b) Ventrículo esquerdo hipertrófico;

c) Afilamento;

d) Cardiomiopatia dilatada;

e) Prolapso da valva mitral.

- Sexo masculino:
a) Ponte miocárdica;
b) Espasmo;
c) Fístula.

Segundo sua localização na árvore coronariana (1), no ventrículo esquerdo (2) ou na valva mitral (3), aquelas 150 anormalidades foram ainda divididas em três grupos (Tabela 4).

Evidencia-se que, das 150 anormalidades, predominaram as presentes na circulação coronariana (63\%), seguidas do ventrículo esquerdo (34\%) e da valva mitral $(3 \%)$

\section{DISCUSSÃO}

O método empregado, isto é, o visual, na análise dos resultados da CINE, teve a mesma aplicabilidade e reprodutibilidade que as apontadas por outros autores ${ }^{16-18}$.

Ao se analisar as anormalidades encontradas, cabe a indagação: qual seria o papel fisiopatológico que

TABELA 3

Somatória das anormalidades segundo a definição angiográfica

\begin{tabular}{llccc}
\hline № & Anormalidade Angiográfica & \multicolumn{2}{c}{ Sexo } & \multicolumn{2}{c}{ Total } \\
de casos
\end{tabular}


Bastos LC, Cunha CLP. Anormalidades Cineangiocoronariográficas em Pacientes com Isquemia Miocárdica à Cintilografia Perfusional e Coronárias sem Lesões Obstrutivas: Estudo Descritivo. Rev Bras Cardiol Invas 2007; 15(1): 52-60.

TABELA 4

Anormalidades agrupadas segundo sua localização

\begin{tabular}{lrllll}
\hline $\mathbf{1}$ - Na Árvore Coronariana & 2 - No Ventrículo Esquerdo & & 3 - Na Valva Mitral & \\
\hline Tortuosidades & 65 & Ventrículo Esquerdo Hipertrófico & 48 & Prolapso Valvar Mitral & 1 \\
Ponte Miocárdica & 16 & & - & & - \\
Afilamento & 14 & Cardiomiopatia Dilatada & - & & - \\
Espasmo & 2 & & $\overline{51}$ & Subtotal & 1 \\
Fístula & 1 & & & \\
Subtotal & 98 & Subtotal & &
\end{tabular}

Total $=150$ anormalidades

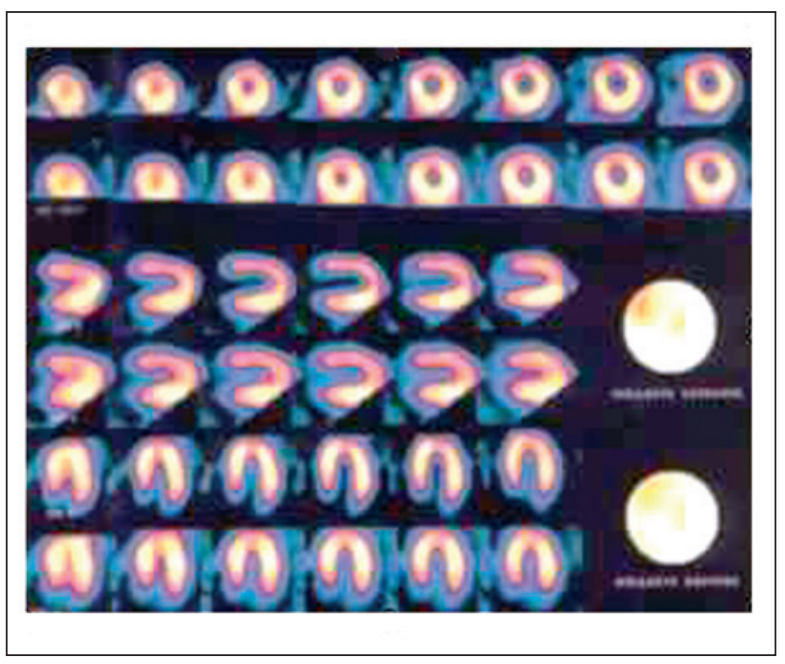

Figura 3 - Conclusão: Cintilografia miocárdica perfusional com Tc $99 m$-Tetrosformin, por técnica tomográfica, compatível com área de hipocaptação parcialmente reversível em região ântero-septal do $V E$, sugestiva de isquemia miocárdica.

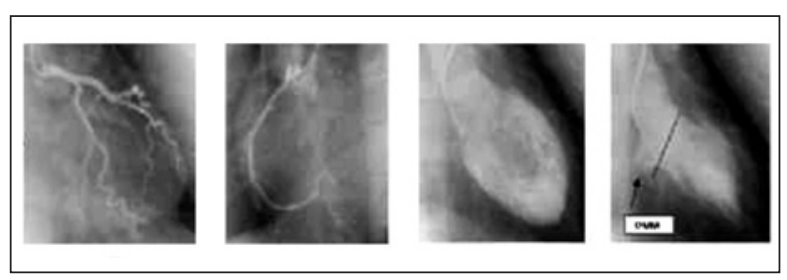

Figura 4 - Circulação coronária sem lesões obstrutivas. Prolapso da valva mitral.

cada uma, isoladamente ou em associação, poderia exercer na gênese da isquemia miocárdica? Procurando respondê-la, é necessário se fazer uma análise das alterações fisiopatológicas que cada uma delas provoca na circulação coronária e que, assim, poderia resultar em uma CPM positiva com CINE sem DAC. Obedecendose à seqüência descrita nas anormalidades angiográficas na seção Método, obtivemos os resultados descritos a seguir.
A anormalidade Tortuosidades foi encontrada isoladamente em 19 pacientes, com significativo predomínio do sexo feminino (15 × 4 casos). Esta freqüência maior de casos no sexo feminino é corroborada por Macruz et al. ${ }^{20}$ que foram os primeiros a considerar a tortuosidade como causa de insuficiência coronária. Segundo estes autores, ela provocaria alongamento, isto é, aumento do comprimento do sistema arterial, transformando o fluxo laminar em turbilhonar, favorecendo, teoricamente, a agregação plaquetária momentânea. Assim, ocorreria, conseqüentemente, aumento da resistência vascular coronária, com diminuição do fluxo e da velocidade no sistema. O estudo anatomopatológico realizado no trabalho publicado por estes autores não revelou obstrução ou alteração nas paredes dos vasos coronários, incluindo os da microcirculação. Portanto, a análise conjunta destes dados levou-os a ponderar ser a tortuosidade a responsável pela isquemia miocárdica. Considerando os achados fisiológicos descritos por estes autores, os mesmos poderiam provocar um resultado falso-positivo, corroborando a presença de CPM positiva nos pacientes do presente trabalho. É importante comentar que este achado foi encontrado mais freqüentemente associado a outros achados do que isoladamente, conforme a Tabela 2 .

O Espasmo foi encontrado em apenas dois pacientes, ambos do sexo masculino, e não de forma isolada. Entretanto, é sabido que o espasmo coronário espontâneo é causa de angina estável clássica na ausência de $\mathrm{DAC}^{28,29}$. A presença desta anormalidade angiográfica nos pacientes sem DAC, comprovada pela CINE, não afasta a presença de aterosclerose no ou próxima ao sítio responsável pelo mesmo. Deve ser lembrado que a presença de pequenas placas ateroscleróticas pode não ser detectada na CINE. Isto foi bem demonstrado por MacAlpin ${ }^{30}$, que encontrou aterosclerose coronária na vizinhança do local afetado pelo espasmo em $88 \%$ dos casos estudados. Como já comentado anteriormente, esta anormalidade angiográfica não foi encontrada isoladamente na presente casuística, somente em associação (Tortuosidades e Ponte Miocárdica) e com poucos casos: apenas dois. Portanto, não se pode afirmar que 
Bastos LC, Cunha CLP. Anormalidades Cineangiocoronariográficas em Pacientes com Isquemia Miocárdica à Cintilografia Perfusional e Coronárias sem Lesões Obstrutivas: Estudo Descritivo. Rev Bras Cardiol Invas 2007; 15(1): 52-60.

ambos sejam casos clássicos de angina vasoespástica. É mais sensato considerá-los como provocados pela presença do cateter. Entretanto, se houve o espasmo, alguma hiper-reatividade vascular deve ter ocorrido nos dois casos. A presença desta anormalidade angiográfica isoladamente pode ser causa de isquemia miocárdica e, portanto, provocar uma CPM positiva, corroborando os nossos achados.

Quanto à presença de Ponte Miocárdica, foram encontrados isoladamente 6 casos, com predomínio do sexo masculino. Entretanto, a mesma foi encontrada em associação em outros 9 casos, com um número maior neste sexo, porém já ocorrendo um aumento na sua presença no sexo feminino. Desde sua definição ${ }^{31}$, o substrato clínico responsável pela sintomatologia anginosa ainda é motivo de divergências. Alguns autores $^{32,33}$ consideram que a compressão transitória da artéria coronária, provocada pela ponte miocárdica, pode estar associada a manifestações clínicas de isquemia miocárdica, durante atividade física intensa. Laurent et al. $^{34}$ questionam apenas a compressão como causa da sintomatologia, uma vez que a perfusão coronária é preferencialmente diastólica. Estes autores sugerem que outros mecanismos estejam envolvidos na gênese das alterações perfusionais, entre eles a taquicardia, o espasmo coronário e a trombose. Corroborando estes dados, vários autores ${ }^{35-39}$, empregando a ultra-sonografia intracoronária em pacientes sintomáticos portadores de ponte miocárdica, demonstraram que a compressão do vaso não está limitada à sístole, mas também persiste na diástole, afetando, portanto, a fase predominante da perfusão coronariana. Além disto, Hongo et al. ${ }^{36}$ demonstraram que o atraso na dilatação do lúmen coronariano, durante o início da diástole, pode contribuir para a ocorrência de isquemia coronariana. Estes dados são fundamentos importantes na gênese da alteração perfusional coronária, podendo justificar CPM positiva na ausência de DAC.

Já o Prolapso da Valva Mitral foi encontrado em apenas um caso, sendo este do sexo feminino. No estudo epidemiológico realizado em Framingham ${ }^{40}$, houve predominância do sexo feminino, principalmente na população mais jovem, corroborando o achado encontrado no presente trabalho. Esta anormalidade angiográfica já era conhecida desde 196131, quando houve a primeira publicação sobre a sua presença em autópsias de indivíduos com idade acima de 40 anos, sendo encontrada em 5\% dos casos estudados. Entretanto, desde então, sua relação com a cardiopatia isquêmica era controversa. Likoff et al. ${ }^{41}$ foram os primeiros a levantar a hipótese de dor torácica acometer pacientes com artérias coronárias normais à cinecoronariografia. Ao analisarem 218 pacientes com prolapso, Wicklife et al. ${ }^{42}$ demonstraram que a presença de DAC é baixa, tendo sido encontrada em apenas $6 \%$ dos seus casos. Além disto, vários autores ${ }^{43-46}$ reconhecem que esta doença vem acompanhada de uma variedade de sintomas inespecíficos, como fadiga, palpitações, tonturas, desconforto torácico e sintomas neuropsiquiátricos, bem como sintomas de disfunção autonômica. Assim, acredita-se que pelo menos parte dos sintomas relacionados ao Prolapso da Valva Mitral seja decorrente da elevada associação com hiperatividade adrenérgica, ansiedade, pânico e astenia neurocirculatória. Outra explicação seria a tensão sobre os músculos papilares, provocada pelo movimento do folheto prolapsado durante a sístole. De qualquer forma, apesar de não se ter ainda a explicação mais fidedigna do desconforto torácico, esta anormalidade angiográfica pode provocar alteração da perfusão na CPM, confirmando o achado de sua positividade na ausência de DAC à CINE.

Considerando-se a presença de Afilamento, esta anormalidade angiográfica não foi encontrada isoladamente nesta casuística, estando sempre associada a outras anormalidades, sendo as mais freqüentes Tortuosidades e Ventrículo Esquerdo Hipertrófico, predominando no sexo feminino. Esta mesma predominância é corroborada por Macruz et al. ${ }^{20}$, que a encontraram em 105 pacientes portadores de Tortuosidades e Afilamento. Segundo estes autores, esta associação acarretaria em aumento da resistência ao fluxo coronário, podendo levar à insuficiência coronária. Este diagnóstico clínico foi demonstrado por meio de manifestações peculiares encontradas nas análises clínica, eletrocardiográfica e CINE daqueles 105 casos. Desta forma, esta anormalidade angiográfica pode ser responsável pela presença de CPM positiva e CINE normal, na presente casuística.

Quanto à Anomalia Congênita Coronária-Fístula, esta anormalidade angiográfica foi encontrada em apenas um paciente do sexo masculino e associada à condição de Ventrículo Esquerdo Hipertrófico. As fístulas coronárias são anomalias $\operatorname{raras}^{47}$ e de significado patológico controverso, exceto naqueles casos onde as mesmas apresentam-se de tamanho e fluxo elevados, podendo provocar não só roubo de fluxo coronário, mas também síndrome de alto débito, com sua manifestação clínica clássica. Porém, a maioria dos pacientes é assintomática. Assim, a expressão clínica de insuficiência coronária ou insuficiência cardíaca é menos freqüen$\mathrm{te}^{48}$. Há relato ${ }^{49}$ da presença de fístula em pacientes com angina de peito, podendo ocorrer esta sintomatologia em até 38\% deles. Assim, esta anormalidade angiográfica também pode provocar alteração perfusional à CPM com coronárias angiograficamente normais.

O Ventrículo Esquerdo Hipertrófico foi encontrado isoladamente em nove pacientes, havendo leve predomínio no sexo masculino. Como bem demonstrado por vários autores ${ }^{50-52}$, esta modificação do fluxo coronário. Segundo eles, isto se deve ao desequilíbrio provocado entre a oferta e a demanda de oxigênio no músculo hipertrofiado e conseqüente alteração, inicialmente, da função diastólica e, mais tardiamente, da função 
Bastos LC, Cunha CLP. Anormalidades Cineangiocoronariográficas em Pacientes com Isquemia Miocárdica à Cintilografia Perfusional e Coronárias sem Lesões Obstrutivas: Estudo Descritivo. Rev Bras Cardiol Invas 2007; 15(1): 52-60.

sistólica. Estas alterações provocadas pela hipertrofia miocárdica são responsáveis por defeitos perfusionais à CPM. Desta forma, este achado angiográfico justificaria a presença de CPM falso-positiva encontrada nos pacientes portadores da mesma, na presente casuística.

A Cardiomiopatia Dilatada ocorreu, isoladamente, em apenas um paciente do sexo masculino e, de forma associada, em dois pacientes do feminino. A ocorrência de dor torácica nos pacientes portadores desta doença é descrita por vários autores ${ }^{21,53-55}$. Apesar da sintomatologia anginosa ser menos freqüente do que a relacionada à disfunção ventricular esquerda, ela é passível de acometer até um terço dos indivíduos, o que poderia ser causa de CPM positiva, na ausência de DAC. Isto justificaria o achado no presente trabalho.

É importante ressaltar que este trabalho foi feito de forma retrospectiva e em apenas uma instituição e, portanto, não deve ser generalizado para outras situações. No entanto, o mesmo analisa uma população heterogênea e do mundo real de um Serviço de Hemodinâmica, refletindo uma realidade encontrada por todos os autores pesquisados. Como, então, explicar uma incidência tão alta de falso-positivos nas CPM? Embora, não se tenha uma resposta única e objetiva a esta questão, deve-se sempre ter em mente que a sensibilidade e a especificidade da CPM são resultantes da comparação com a $\mathrm{CINE}^{47}$. Isto significa dizer que a primeira avalia o fluxo coronário, enquanto a segunda analisa apenas a anatomia macroscópica das artérias coronárias. Portanto, a presença de lesão na CINE não reflete, necessariamente, o grau de comprometimento do fluxo arterial coronário ${ }^{13}$.

O Consenso SOCESP-SBC ${ }^{56}$ sobre Medicina Nuclear considera as discrepâncias entre a CPM e a CINE resultantes da baixa correlação entre o grau de estenose coronária e a gravidade funcional avaliada durante a determinação do fluxo coronário, obtido na vasodilatação farmacológica máxima. Além disto, vários autores ${ }^{9,57-60}$ chamam a atenção para o fato de que o significado dos resultados dos testes relaciona-se não somente com a sensibilidade e a especificidade, mas também com a prevalência da doença na população submetida aos mesmos (Teorema de Bayes). Nesta linha de raciocínio, se o teste é realizado numa população de baixo risco para DAC, a chance de um resultado falso-positivo é maior que o encontrado em uma população de alto risco. Isto é corroborado pela Sociedade Brasileira de Cardiologia, em suas recém-publicadas "Diretrizes de Doença Coronariana Crônica - Angina Estável"61, ao sugerir que "na avaliação da estratificação de risco, é fundamental que o cardiologista tenha noções da seqüência bayesiana, onde conhecimentos da probabilidade pré-teste da DAC, associados à sensibilidade e à especificidade dos métodos não invasivos, possibilitam estabelecer uma adequada interpretação dos resultados, bem como a melhor estratégia".

\section{CONCLUSÃO}

Foram identificadas as seguintes anormalidades angiográficas na CINE:
a) tortuosidade;
b) espasmo;
c) ponte miocárdica;
d) prolapso da valva mitral;
e) afilamento;
f) fístula coronária congênita anômala;
g) ventrículo esquerdo hipertrófico;
h) cardiomiopatia dilatada.

Todas elas, em maior ou menor grau, isoladas ou em associação, podem ser responsáveis por defeitos de perfusão miocárdica e, portanto, passíveis de originar CPM "falso-positivas" com artérias coronárias sem lesões obstrutivas. Embora não se possa afirmar que aquelas anormalidades angiográficas sejam a causa direta desta discrepância, elas possivelmente contribuíram para esta ocorrência.

Diante do exposto, nas populações de baixo risco ou de risco intermediário, principalmente em mulheres, deve-se fazer uma triagem dos pacientes candidatos à CPM, procurando afastar doenças passíveis de serem detectadas por outros métodos não invasivos, como a ecodopplercardiografia, para afastar a presença de prolapso da valva mitral, ventrículo esquerdo hipertrófico e cardiomiopatia dilatada. Naqueles pacientes portadores de tortuosidade, afilamento, fístula coronária congênita anômala e ponte miocárdica, já podem ser utilizados, em grandes centros médicos, os métodos não invasivos mais sofisticados, como a tomografia computadorizada multislice e a tomografia computadorizada por feixe de elétrons, que têm apresentado excelentes resultados na identificação destas anormalidades ${ }^{62-64}$. Finalmente, é necessário deixar registrado que a CINE é o padrãoouro para a avaliação da precisão diagnóstica dos métodos que estudam a isquemia miocárdica, nos casos de suspeita da presença de DAC.

\section{AGRADECIMENTOS}

Nossos agradecimentos aos Srs. Drs. Evandro Luis Queiros Flores e Álvaro Campos, pelo apoio nas viagens a Curitiba e levantamento dos laudos e imagens das cintilografias, respectivamente, e aos Srs. Drs. Marcelo Dias Carneiro e Danton Richilin Rocha Loures, pela correção do Summary e co-orientação durante a elaboração e conclusão deste trabalho, respectivamente.

\section{REFERÊNCIAS BIBLIOGRÁFICAS}

1. Who Monica Project. Myocardial Infarction and Coronary Deaths in the World Health Organization Monica Project. Circulation 1994;90:583-616.

2. Lotufo PA. Epidemiologia das doenças cardíacas no Brasil: histórico, situação atual e proposta de modelo teórico. Rev Soc Cardiol Est São Paulo 1996;6:541-7. 
Bastos LC, Cunha CLP. Anormalidades Cineangiocoronariográficas em Pacientes com Isquemia Miocárdica à Cintilografia Perfusional e Coronárias sem Lesões Obstrutivas: Estudo Descritivo. Rev Bras Cardiol Invas 2007; 15(1): 52-60.

3. Lotufo PA. Mortalidade precoce por doenças do coração no Brasil; comparação com outros países. Arq Bras Cardiol 1998; 70:321-5.

4. Buss PM. Assistência hospitalar no Brasil (1984-1991): uma análise preliminar baseada no Sistema de Informação Hospitalar do SUS. Inf Epidemiol SUS 1993;2:5-44.

5. Carr EA, Gleason G, Shaw J, Krontz B. The direct diagnosis of myocardial infarction by photoscanning after administration of cesium-131. Am Heart J 1964;68:627-36.

6. Beller GA. Clinical nuclear cardiology. Philadelphia:WB Saunders;1995. p.82-136.

7. Feitosa GS, Nicolau JC, Chalela WA, Meneghetti JC, Ximenes $A A B$, Almeida CA, et al. I Diretriz da Sociedade Brasileira de Cardiologia sobre Cardiologia Nuclear. Arq Bras Cardiol 2002;78(supl.III):5-42.

8. Beller GA. Current status of nuclear cardiology techniques. Curr Probl Cardiol 1991;16:449-535.

9. Berman DS, Hachamovitch R, Kiat H, Cohen I, Cabico JA, Wang PF, et al. Incremental value of prognostic testing in patients with known or suspected ischemic heart disease: a basis for optimal utilization of exercise technetium-99m sestamibi myocardial perfusion single-photon emission computed tomography. J Am Coll Cardiol 1995;26:639-47.

10. Beller GA, Zaret BL. Contributions of nuclear cardiology to diagnosis and prognosis of patients with coronary artery disease. Circulation 2000;101:1465-78.

11. Hironaka FH. Medicina nuclear em coronariopatia crônica, cardiopatia isquêmica: aspectos de importância clínica. São Paulo:Sarvier;1989. p.345-57.

12. Meneghelo RS. Teste ergométrico, cintilografia e cinecoronariografia: correlação e discrepâncias. Rev Soc Cardiol Est São Paulo 1995;3:285-9.

13. White CW, Wright CB, Doty DB, Hiratza LF, Eastham CL, Harrison DG, et al. Does visual interpretation of the coronary arteriogram predict the physiologic importance of a coronary stenosis? N Engl J Med 1984;310:810-24.

14. Fleming RM, Kirkeeide RL, Smalling RW, Gould KL. Patterns in visual interpretation of coronary arteriograms as detected by quantitative coronary angiography. J Am Coll Cardiol 1991;18:945-51.

15. Stadius ML, Alderman EL. Coronary artery revascularization: critical need for, and consequences of objective angiographic assessment of lesion severity. Circulation 1990;82:2231-4.

16. DeRouen TA, Murray JA, Owen W. Variability in the analysis of coronary arteriograms. Circulation 1977;55:324-8.

17. Guimarães JA, Victor EG, Leite MRB, Gomes JM, Victor Filho E, Reyes Liveras J. Confiabilidade da interpretação da cinecoronariografia pelo método visual simples. Arq Bras Cardiol 2000;74:291-9.

18. Kussmaul WG 3rd, Popp RL, Norcini J. Accuracy and reproducibility of visual coronary stenosis using estimates information from multiple observers. Clin Cardiol 1992;15:154-62.

19. Baumgart D, Schmermund A, George G, Haude M, Ge J, Adamzik $M$, et al. Comparison of electron beam computed tomography with intracoronary ultrasound and coronary angiography for detection of coronary atherosclerosis. J Am Coll Cardiol 1997;30:57-64.

20. Macruz R, Toriano N, Arie S, Godoy M, Carvalho VB, Armelin $\mathrm{E}$, et al. Síndrome da insuficiência coronária não obstrutiva. Tortuosidades das artérias coronárias. Arq Bras Cardiol 1976;29:255-62.

21. Coughlin SS, Comstock GW, Baughman KL. Descriptive epidemiology of idiopathic dilated cardiomyopathy in Washington County, Maryland, 1975-1991. J Clin Epidemiol 1993;46:1003-8.
22. Carvalho VB, Macruz R, Decourt LV, Arie S, Manrique R, Mello SC, et al. Hemodynamic determinants of coronary constriction in human myocardial bridges. Am Heart J 1984; 108:73-80.

23. Jeresaty RM. Mitral valve prolapse: definition and implications in athletes. J Am Coll Cardiol 1986;7:231-6.

24. Campos ERS. Anomalias congênitas das artérias coronárias: incidência e classificação: estudo angiográfico [Dissertação]. São Paulo:Faculdade de Medicina da Universidade de São Paulo;1981.

25. Aloan L. Hemodinâmica e angiocardiografia: obtenção de dados, interpretação, aplicações clínicas. $2^{a}$ ed. Rio de Janeiro:Atheneu;1990. p.498-510.

26. Sones FM Jr. Acquired heart disease: Symposium on Present and Future Cineangiocardiography. Am J Cardiol 1959;3:710.

27. Judkins MP. Selective coronary arteriography. I a percutaneous transfemoral technic. Radiology 1967;89:815-24.

28. Boden WE, Bough EW, Korr KS, Benham I, Gheorghiade M, Caputi A, et al. Exercise-induced coronary spasm with S-T segment depression and normal coronary arteriography. Am J Cardiol 1981;48:193-7.

29. Chahine RA. The diagnosis coronary artery spasm in the cardiac catheterization laboratory. Cardiol Clin 1985;3:19-28.

30. MacAlpin RN. Relation of coronary arterial spasm to sites of organic stenosis. Am J Cardiol 1980;46:143-53.

31. Polacek $\mathrm{P}$, Kralove $\mathrm{H}$. Relation of myocardial bridges and loops on the coronary arteries occlusions. Am Heart 1961;61:44-52.

32. Bestetti RB, Costa RS, Kazava DK, Oliveira JS. Can isolated myocardial bridging of the left anterior descending coronary artery be associated with sudden death during exercise? Acta Cardiol 1991;46:27-30.

33. Kracoff $\mathrm{OH}$, Ovsyshcher I, Gueron M. Malignant course of a benign anomaly: myocardial bridging. Chest 1987;92:1113-5.

34. Laurent G, Cottin Y, Andre F, Pichon E, Piszker G, Gerard C, et al. Ponts myocardiques symptomatiques. À propos de 6 cas. Arch Mal Coeur 1996;89:883-7.

35. Ge J, Jeremias A, Rupp A, Abels M, Baumgart D, Liu F, et al. New signs characteristic of myocardial bridging demonstrated by intracoronary ultrasound and Doppler. Eur Heart J 1999;20:1707-16

36. Hongo $\mathrm{Y}$, Tada $\mathrm{H}$, Ito K, Yasumura $\mathrm{Y}$, Miyatake $\mathrm{K}$, Yamagishi $M$. Augmentation of vessel squeezing at coronary-myocardial bridge by nitroglycerin: study by quantitative coronary angiography and intravascular ultrasound. Am Heart J 1999; 138(2pt1):345-50.

37. Jain SP, White $C$ J, Ventura HO. De novo appearance of a myocardial bridge in heart transplant: assessment by intravascular ultrasonography, doppler and angioscopy. Am Heart J 1993;126:453-6.

38. Schwarz ER, Klues HG, vom Dahl J, Klein I, Krebs W Hanrath P. Functional, angiographic and intracoronary doppler flow characteristics in symptomatic patients with myocardial bridging: effect of short-term intravenous beta-blocker medication. J Am Coll Cardiol 1996;27:1637-45.

39. Winter RJ, Kok WE, Piek JJ. Coronary atherosclerosis within a myocardial bridge, not a benign condition. Heart 1998; 80:91-3.

40. Savage DD, Garrison RJ, Devereux RB,Castelli WP, Anderson SJ, Levy D, et al. Mitral valve prolapse in the general population. 1. Epidemiologic features: The Framingham Study. Am Heart J 1982;106:571-6.

41. Likoff W, Segal BL, Kasparian H. Paradox of normal selective coronary arteriograms in patients considered to have unmistakable coronary heart disease. N Engl J Med 1967;276: 1063-6. 
Bastos LC, Cunha CLP. Anormalidades Cineangiocoronariográficas em Pacientes com Isquemia Miocárdica à Cintilografia Perfusional e Coronárias sem Lesões Obstrutivas: Estudo Descritivo. Rev Bras Cardiol Invas 2007; 15(1): 52-60.

42. Wicklife C. The coronary circulation in idiopathic mitral valve prolapse. Circulation 1974;50(supl.3):11-7.

43. Boudoulas H, Kolibas AJ Jr, Baker P, King BD, Woole CF. Mitral valve prolapse and the mitral valve prolapse syndrome: a diagnostic, classification and pathogenesis of symptoms. Am Heart J 1989;118:796-818.

44. Davies AO, Mares A, Pool Jl, Taylor AA. Mitral valve prolapse with symptoms of beta-adrenergic hypersensisitivity. Beta 2-adrenergic receptor supercoupling with desensibilization on isoproterenol exposure. Am J Med 1987;82:193-201.

45. Gaffney FA, Bastian BC, Lane LB, Taylor WF, Horton J, Schutte $\mathrm{JE}$, et al. Abnormal cardiovascular regulation on the mitral valve prolapse syndrome. Am J Cardiol 1983;52:316-20.

46. Pudu PE, Pasternac A, Tubau JF, Krol R, Farley L, de Champlian J. QT interval prolongation and increased plasma catecholamine levels in patients with mitral valve prolapse. Am Heart J 1983;105:422-8.

47. Carvalho VB, Macruz R. Cardiopatia isquêmica: aspectos de importância clínica. São Paulo:Sarvier;1989. p.255-80.

48. Yamanaka O, Hobbs RE. Coronary artery anomalies in 126,595 patients undergoing coronary arteriography. Cathet Cardiovasc Diagn 1990;21:28-40.

49. Liberthson RR, Sagar K, Berkoben JP, Weintraub RM, Levine $\mathrm{FH}$. Congenital coronary arteriovenous fistula. Report of 13 patients, review of the literature and delineation of management. Circulation 1979;59:849-54.

50. Ghali JK, Kadakia S, Cooper RS, Liao YL. Impact of left ventricular hypertrophy on ventricular arrythmias in the absence of coronary artery disease. J Am Coll Cardiol 1991; 17:1227-82.

51. Levy D, Anderson KM, Savage DD, Kannel WB, Christiansen JC, Castelli WP, et al. Echocardiographycally detected left ventricular hypertrophy: prevalence and risk factors. The Framingham Heart Study. Ann Intern Med 1988;108:7-13.

52. Widgren BR, Persson B, Wikstrand J. Reduced left ventricular distensibility in normotensive men with a positive family history of hypertension. Am J Hypertens 1993;6:750-7.
53. Dec GW, Fuster V. Idiopathic dilated cardiomyopathy. N Engl J Med 1994;331:1564-75.

54. Komajda M, Jais JP, Reeves F, Goldfarb B, Bouhour JB, Juillieres $\mathrm{Y}$, et al. Factors predicting mortality in idiopathic dilated cardiomyopathy. Eur Heart J 1990;11:824-31.

55. Sugrue DD, Rodeheffer RJ, Codd MB, Ballard DJ, Fuster V, Gersh BJ. The clinic course of idiopathic dilated cardiomyopathy. A population-based study. Ann Inter Med 1992;117: 117-23.

56. Camargo EE, Marin-Neto JA, Naccarato AF, Ramires JA, de Castro I, Paiva EV, et al. Consenso SOCESP-SBC sobre medicina nuclear. Arq Bras Cardiol 1995;5:469-74.

57. Andrade PJN. Sistemas especialistas de apoio ao diagnóstico em medicina. Relações com o teorema de Bayes e com a lógica do raciocínio diagnóstico. Arq Bras Cardiol 1999;73: 537-43.

58. Ritchie JL. Guidelines for clinical use of cardiac radionuclide imaging. Circulation 1995;91:1278-303.

59. Verna E, Ceriani L, Giovanella L, Binaghi G, Garancini S. "False-positive" myocardial perfusion scintigraphy findings in patients with angiographically normal coronary arteries: insights from intravascular sonography studies. J Nucl Med 2000;4:1935-40.

60. Wagner HN Jr. Baye's theorem: an idea whose time has come? Am J Cardiol 1982;49:875-7.

61. Mansur AP, Armaganijan DA, Sousa JG, Simão AC, Brito $\mathrm{AF}$, Xavier A, et al. Diretrizes de doença coronariana crônica - angina. Arq Bras Cardiol 2004;83(supl.2):2-43.

62. Cademartiri F, Mollet N, Nieman K, Szili-Torok T, de Feyter PJ. Right coronary artery arising from the left circumflex demonstrated with multislice computed tomography. Circulation 2004;109:e185-6.

63. Möhlenkamp S, Hort W, Ge J, Erbel R. Update on myocardial bridging. Circulation 2002;106:2616-22.

64. Goo HW, Park IS, Ko JK, Kim YH, Seo DM, Yun TJ, et al. $\mathrm{CT}$ of congenital heart disease: normal anatomy and typical pathologic conditions. Radiographics 2003;23:S147-65. 